Antiepileptic drugs have variable effects on attention and behavior. Phenobarbital, gabapentin, and topiramate cause deterioration, whereas carbamazepine and lamotrigine have been shown to improve attention and behavior. Phenytoin and oxcarbazepine have no documented effect on symptoms of ADHD. Concern that stimulant medications, methylphenidate (MPH) and dextroamphetamine (DAM), may lower the seizure threshold are not supported by controlled studies. In fact, most studies show that seizure frequency is not increased by MPH in children with controlled epilepsy (one study found an increased risk of a seizure with MPH in ADHD children with epileptiform EEGs but no previous seizure). DAM has been used as adjunct therapy in the control of nocturnal seizures. Atomoxetine and clonidine have no reports of seizure induction, but buproprion carries a dose-related risk of seizures. (Schubert R. Attention deficit disorder and epilepsy. Pediatr Neurol January 2005;32:1-10). (Respond: Dr Romaine Schubert, Chief, Division of Pediatric Neurology, New York Methodist Hospital. $5036^{\text {th }}$ St, Rm 518, East Pavilion, Brooklyn, NY 11215).

COMMENT. This review of ADHD and epilepsy is supported by 108 references and provides a scientific basis for the management of children with this common combination of symptoms. One in 5 children with epilepsy may have ADHD (Gross-Tsur V et al. J Pediatr 1997;130:670-674). Further studies are required, especially regarding the significance of subclinical epileptiform discharges in the EEG of children with ADHD and "staring" or "daydreaming" episodes, and the question "to treat or not to treat" with AEDs.

That MPH is effective and safe in children with ADHD and epilepsy, well controlled with antiepileptic drugs, is supported by another current review (Tan M, Appleton R. Attention deficit and hyperactivity disorder, methylphenidate, and epilepsy. Arch Dis Child Jan 2005;90:57-59). In contrast, children with ADHD and epileptiform EEGs may develop seizures with the introduction of MPH (Hemmer SA et al. Pediatr Neurol 2001;24:99-102). The incidence of seizures with MPH in those children with ADHD complicated by centrotemporal (rolandic) spikes was $16.7 \%$, compared to only $0.6 \%$ in the group with normal EEGs. Other studies have shown either no effect or a reduction of seizures with MPH in children with ADHD and abnormal EEG or epilepsy.

\title{
PHYSICIAN FOLLOW-UP CARE OF CHILDREN WITH ADHD
}

Follow-up care received by children with attention deficit hyperactivity disorder ( $\AA D H D)$ by primary care clinicians (PCCs) was evaluated by questionnaires completed by parents at an index visit and at six months, in a study at Ohio State University, Columbus, $\mathrm{OH}$ and several research networks. Each clinician enrolled a consecutive sample of 55 children, 4 to 15 years of age, and 976 children identified with ADHD were selected for follow-up. Surveys were returned by $659(68 \%)$ families, and the outcome measure was the number of office visits during the 6 months. Medications ( $94 \%$ stimulants) were prescribed at the index visit in $52 \%$ children with ADHD, and $78 \%$ were medicated at 6 months. A median of one visit was made to the PCC in 6 months, and the number of visits was the same for those taking psychotropic medication as those not on medication. Follow-up visits were more frequent to PCCs who had completed a fellowship in mental health training. Children seeing a mental health specialist $(26 \%)$ were more often black, on Medicaid, or had higher levels of internalizing symptoms. Follow-up care by PCCs for ADHD falls below that recommended 
in the 2000-1 AAP Guidelines. (Gardner W, Kelleher KJ, Pajer K, Campo JV. Follow-up care of children identified with ADHD by primary care clinicians: a prospective cohort study. J Pediatr December 2004;145:767-771). (Reprints: Dr Gardner, Office of Clinical Science, Children's Research Institute, 700 Children's Drive, Suite 1401, Columbus, OH 43205).

COMMENT. Follow-up of children with ADHD requiring medication monitoring is time consuming, and optimal management should include both parent and teacher reports regarding educational and behavioral progress, provided monthly. A psychological evaluation is essential in patients whose academic achievement falls short of that expected. A single clinician visit each 6 months is generally insufficient, and collaboration with a mental health or neurology specialist can optimize results of therapy.

A current clinical practice review and guidelines for diagnosis and management of ADHD, as followed in the Department of Pediatrics and Human Development, Michigan State University, East Lansing, MI, recommends visits every 3 to 4 months, once a stable dose of medication is established (Rappley MD. N Engl J Med January 13, 2005;352:165173). An opportunity to monitor medication between quarterly visits is also afforded by parental telephone requests for prescription renewal at monthly intervals.

Recent warnings regarding rare but serious adverse events with Adderall XR and Strattera (atomoxetine) have emphasized the importance of close medication monitoring to avoid toxicity: patients with a history or signs of cardiac problems should not be treated with Adderall, and patients receiving Strattera should be monitored for symptoms or signs of liver dysfunction.

The physician's role in the collaborative community care of ADHD is outlined in a report of a consensus developed among health care providers and educators in North Carolina Counties (Foy JM, Earls MF. A process for developing community consensus regarding the diagnosis and management of attention-deficit/hyperactivity disorder. Pediatrics Jan 2005;115:e97-e104). The MD's role includes close collaboration with school personnel, and use of communication forms to share diagnostic and medication information with school and family. Large discrepancies were identified between pediatricians' practice patterns and AAP guidelines, and $50 \%$ of children with $\mathrm{ADHD}$ were unidentified and untreated.

\section{HEADACHE DISORDERS}

\section{PRACTICE GUIDELINES FOR MANAGEMENT OF MIGRAINE}

The literature (166 articles identified and reviewed) on the pharmacological treatment of the child with migraine headache was classified according to acute headache and preventive medications, and the results of drug trials were evaluated by Committees of the Child Neurology Society and American Academy of Neurology. Five agents reviewed for acute treatment were evaluated as follows: sumatriptan nasal spray and ibuprofen - effective and well tolerated compared to placebo; acetaminophen - probably effective and well tolerated cf placebo; rizatriptan and zolmitriptan - safe and well tolerated but not superior to placebo. Twelve agents for preventive therapy: flunarizine - probably effective; insufficient data concerning cyproheptadine, amitryptyline, divalproex sodium, topiramate. and 